\title{
Characterization of the effects of multi-tap filtering on FBMC/OQAM systems
}

\author{
Marius Caus ${ }^{1 *}$, Ana I Perez-Neira ${ }^{1,2}$ and Adrian Kliks $^{3}$
}

\begin{abstract}
The filter bank multicarrier modulation based on offset quadrature amplitude modulation (FBMC/OQAM) is being considered as an eligible technology for future wireless communications. However, the orthogonality may be destroyed in the presence of multipath fading, and thus, the demodulated data may be affected by inter-symbol and inter-carrier interference. To restore the orthogonality, it is deemed necessary to either precode the symbols to be transmitted or equalize the demodulated data. Under severe propagation conditions, both the precoders and equalizers should perform multi-tap filtering. In this paper, we try to determine whether it is the best choice to combat the channel at the transmit, or at the receive side. The answer to this question is not trivial, since with data precoding, the transmit power may increase, while equalization at the receive side may enhance the noise power. To cast light on this issue, this paper characterizes the average transmit and noise power to determine the influence of multi-tap filtering on the transmit signal power and on the noise variance. The analysis conducted in this paper reveals that if multi-tap precoding yields a power increase, then the noise variance would increase with the same magnitude if the same linear filter was moved from the transmit to the receive side. Therefore, if symbols are properly scaled when the transmit power increases, there is no degradation due to placing the complexity burden at the reception rather than at the transmission. To scale the symbols, it is mandatory to know the additional transmit power. In this sense, a low-complexity method to estimate the power increase is proposed. Simulation-based results confirm that the estimation is reasonably accurate.
\end{abstract}

\section{Introduction}

Networks are evolving in such a way that different systems with specific characteristics co-exist in the same area. Cognitive radio networks, where secondary users share the same spectrum as the primary licensed users, are a good example. In these systems, secondary users have to detect the spectrum conditions to transmit on those bands where the primary user is inactive [1]. In this regard, there are initiatives to use spectral slots in the professional mobile radio (PMR) band and white spaces freed up by the current digital television system. In these scenarios, the devices that transmit in the unoccupied bands have to guarantee that no interference will be induced in legacy primary networks. Therefore, future wireless communications should be able to transmit in a fragmented spectrum where different spectral components are unlikely to be

\footnotetext{
*Correspondence: marius.caus@upc.edu

${ }^{1}$ Department of Signal Theory and Communications, Universitat Politecnica

de Catalunya (UPC), Barcelona 08034, Spain

Full list of author information is available at the end of the article
}

tightly synchronized. This observation highlights that it is deemed necessary to utilize spectrally agile waveforms, and thus, further research beyond the established orthogonal frequency division multiplexing (OFDM) technique is required [2]. It is worth mentioning that due to the aforementioned features, other modulation formats are also considered for the next generation systems $[3,4]$.

The filter bank multicarrier modulation based on offset quadrature amplitude modulation (FBMC/OQAM), also known as OFDM/OQAM, is a potential candidate to satisfy the upcoming needs of future wireless communications [5]. This modulation is gaining momentum since it achieves maximum bandwidth efficiency as no redundancy is transmitted. In addition, the data transmitted on each subcarrier can be shaped with frequency well-localized waveforms, which allow a flexible use of the spectrum [6]. An efficient implementation of the FBMC/OQAM scheme for non-contiguous spectrum use, which is especially relevant in cognitive radio networks, is presented in [7].

\section{是 Springer}

(c) 2014 Caus et al : licensee Springer. This is an Open Access article distributed under the terms of the Creative Commons

Attribution License (http://creativecommons.org/licenses/by/2.0), which permits unrestricted use, distribution, and reproduction in any medium, provided the original work is properly credited. 
In summary, FBMC/OQAM exhibits a low out-of-band emission while the spectral efficiency is not degraded, which is a desirable feature to protect legacy users in cognitive radio networks or when perfect synchronization between nodes cannot be attained. However, the FBMC/OQAM transmit signal induces interference, which is known as modulation-induced interference or intrinsic interference. Under ideal propagation conditions, the receiver can recover the data perfectly if the subband pulses fulfill the perfect reconstruction (PR) property [5]; thus, interference is eliminated. It must be mentioned that multipath fading destroys this property, and as a consequence, inter-symbol interference (ISI) and inter-carrier interference (ICI) is present at detection. This highlights that the channel has to be counteracted to restore the orthogonality. It is well known that in general, multi-band processing is required to better cope with the interference coming from adjacent subcarriers. On the negative side, the complexity is substantially increased when subcarriers are jointly processed. The complexity order is $\mathcal{O}\left(L_{s} L_{t}\right)$ when each subchannel is equalized after combining the information received in $L_{s}$ adjacent subcarriers through a multi-band processor that uses $L_{t}$ taps per subband. As $L_{s}$ increases, the number of arithmetic operations required to counteract the channel increases as well. Thanks to the good spectral confinement exhibited by the subcarrier signals, the performance enhancement brought by the joint processing of $L_{s}=3$ subcarriers is marginal with respect to the case that $L_{s}=1$ [8]. The improvement could be more significant by increasing $L_{s}$ beyond 3 , yet the complexity would substantially augment. For this reason, we discard the implementation of multi-band equalizers, and we focus on filters that equalize the channel on a per-subcarrier basis. Some examples can be found in the literature when the channel is compensated at reception [9-14] or at the transmit side [15].

When the frequency selectivity of the channel becomes appreciable at the subcarrier level, both the equalizers and the precoders have to perform multi-tap filtering to compensate the channel. Otherwise, orthogonality is destroyed and residual interference terms may significantly degrade the performance. Among all the possible designs, we favor the subband processing based on the frequency sampling approach described in [9] because it offers a good trade-off between performance and implementation complexity. As the authors highlight in [16], the complexity needed to design the optimum minimum mean square error (MMSE) equalizer, which is designed in the time domain [10], is higher while the bit error rate (BER) is only lower than that achieved by the frequency sampling method at a very high energy-bit-to-noise ratio in highly frequency-selective channels [8]. It is important to mention that the equalizer in [9] can be applied at the transmitter as it is proposed in [15].
This paper studies the impact of multi-tap filtering in FBMC/OQAM systems to determine whether it is the best choice to combat the channel at the transmit or at the receive side. A similar comparison has been made in narrowband multiple-input-multiple-output (MIMO) systems by examining different types of linear processing [17]. Therein, the authors have concluded that the receive filters outperform the transmit filters for low signal-to-noise ratio (SNR), while the transmit filters provide better results for high SNR. The conclusions drawn in [17] may not necessarily be the same in this paper because of the characteristics of FBMC/OQAM together with the fact that we propose to apply the same processing either at transmission or at reception. Regarding the FBMC/OQAM specificities, it is important to remark that the FBMC/OQAM signal structure may be responsible for boosting the power when multi-tap precoders are used. By analogy, multi-tap equalization may result in noise enhancement. These two features highlight the necessity of conducting a new analysis, taking into account the FBMC/OQAM characteristics. Based on that, the contributions of the paper may be summarized as follows:

- The average transmit power has been characterized when the channel is pre-equalized at transmission.

- The average noise power has been characterized when the channel is equalized at reception.

- A low-complexity method to estimate the additional transmit power is devised when precoders are designed according to the frequency sampling approach.

From the closed-form expressions derived in this paper, we may conclude that if the transmit power increases/ decreases, then the noise variance increases/decreases as well with the same magnitude, as long as the same filters are used as precoders or equalizers. This reveals that if the transmitted symbols are properly scaled when the transmit processing boosts the power, then there is no degradation due to equalizing the demodulated data instead of precoding the symbols to be transmitted. In view of this discussion, it is of paramount importance to determine if the power is boosted due to transmit processing operations and to state if some normalization is required. The analysis that has been conducted allows us to approximately formulate the power as the function of the statistical expectation of precoders, which depends on the statistical channel information. Thus, a priori knowledge of channel statistics is required. It is worth mentioning that many algorithms, such as the MMSE channel estimation, also make use of channel statistics information [18]. Alternatively, we may compute the instantaneous transmit power given the precoders. However, the power has to be recalculated if precoders are updated to adapt 
to the new channel conditions. Simulation-based results confirm that the method based on the statistical channel information characterizes the transmit power with reasonable accuracy. Hence, the proposed method may be used to determine if the transmitted symbols should be scaled or not without computing the instantaneous power, thus reducing the complexity.

The rest of the paper is organized as follows. Section 2 describes a FBMC/OQAM-based communication system where channel impairments are combated either at the transmission or at the reception side. Next, Section 3 addresses the design of equalizers and precoders, which may have multiple taps. The effects of performing multitap filtering are studied in Section 4. To that end, we provide analytical expressions to determine if multi-tap precoding boosts the average transmit power and if multitap equalization enhances the average noise power. In order to validate the closed-form expressions derived in Section 4, some numerical results are presented in Section 5. Finally, Section 6 draws the conclusions.

\section{System model}

This section is devoted to describing the communication system that is depicted in Figure 1, which is based on the FBMC/OQAM scheme [5]. It should be noted that at the transmit side, the sequence of low-rate symbols $\left\{x_{m}[k]\right\}$ is divided into $M$ branches. At each branch, the input symbols are linearly filtered by multi-tap precoders $\left\{b_{m}[k]\right\}$. Then, the precoded symbols are fed into the synthesis filter bank (SFB) to perform multiplexation in the frequency domain. The block diagram of the transmitter highlights that the SFB consists of a bank of filters that is built upon the prototype pulse $p[n]$. In other words, the subband filters are generated by frequency shifting $p[n]$. The SFB output may be regarded as the superposition of $M$ subcarrier signals in time, which is formulated as

$$
s_{\mathrm{tr}}[n]=\sum_{k \in \mathbb{Z}} \sum_{m=0}^{M-1}\left(x_{m}[k] * b_{m}[k]\right) f_{m}\left[n-k \frac{M}{2}\right],
$$

where $f_{m}[n]=p[n] e^{j \frac{2 \pi m}{M}(n-(L-1) / 2)}$. The prototype pulse is a finite impulse response filter of the order $L-1$. The delay $\frac{L-1}{2}$ forces the subband filter to be causal [5]. In this paper, the pulse $p[n]$ is designed by following the frequency sampling approach proposed in [19] with an overlapping factor equal to four, thus $L=4 M$. The prototype pulse is properly scaled to have unit energy, i.e., $\sum_{\forall n}|p[n]|^{2}=1$. Note that due to the rate conversion, different sampling indexes have been used. In this sense, the index $n$ is used by high-rate signals, while $k$ is utilized by low-rate signals. It is important to remark that the transmitted symbols are generated by staggering real and imaginary parts of $M_{s}$-ary QAM symbols. Therefore, the symbols may be represented in the format $x_{m}[k]=$ $d_{m}[k] \theta_{m}[k]$, where $d_{m}[k]$ is drawn from a $\sqrt{M_{s}}$ ary pulseamplitude modulation (PAM) constellation, and the phase term is defined as

$$
\theta_{m}[k]=\left\{\begin{array}{ll}
1 & k+m \text { even } \\
j & k+m \text { odd }
\end{array} .\right.
$$

At the other end of the link, the transmitted signal is affected by multipath propagation and contaminated by noise. As a consequence, the received signal is given by $r[n]=h[n] * s_{\mathrm{tr}}[n]+v[n]$, where $h[n]$ is the channel impulse response (CIR), and $v[n]$ is the sequence that contains the noise samples. The noise at the receiver input is circularly symmetric and Gaussian distributed, i.e., $v[n] \sim$ $\mathcal{C N}\left(0, N_{0}\right)$. To recover the information conveyed on each subband, the received samples are fed into a bank of filters, which are matched to the transmit filters, and then the outputs are downsampled. The output of the $q$ th filter is given by $y_{q}[k]=\left(r[n] * f_{q}^{*}[-n]\right)_{\downarrow \frac{M}{2}}$. The operation (.) $\downarrow x$ performs a decimation by a factor of $x$. To combat the channel impairments at the receive side, demodulated signals can be processed with multi-tap equalizers $\left\{a_{q}[k]\right\}$. At the output of the $q$ th equalizer, we receive an estimation of the transmitted data that is written as

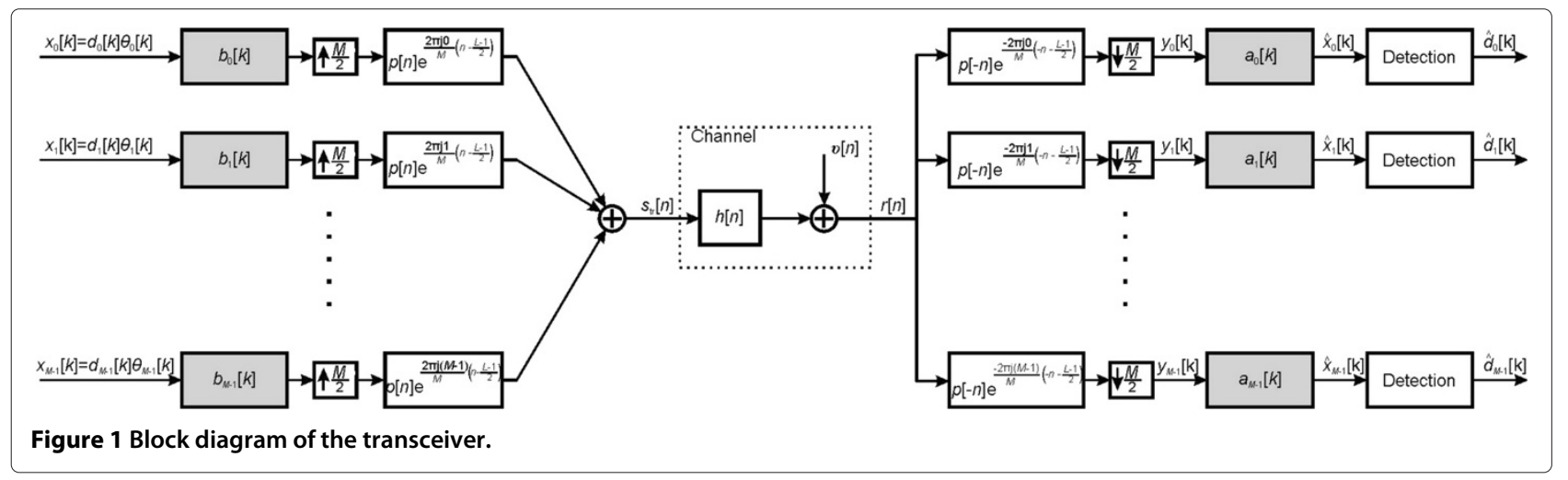




$$
\begin{aligned}
\hat{x}_{q}[k]= & a_{q}[k] * y_{q}[k]=\sum_{m=q-1}^{q+1} a_{q}[k] *\left(d_{m}[k] \theta_{m}[k]\right) * b_{m}[k] \\
& * g_{q m}[k]+a_{q}[k] *\left(v[n] * f_{q}^{*}[-n]\right)_{\downarrow \frac{M}{2}}
\end{aligned}
$$

with

$$
g_{q m}[k]=\left(f_{m}[n] * h[n] * f_{q}^{*}[-n]\right)_{\downarrow \frac{M}{2}} .
$$

Since the frequency spectrum of $p[n]$ is confined within the interval $\left[\begin{array}{ll}-\frac{2 \pi}{M} & \frac{2 \pi}{M}\end{array}\right]$, the subcarrier signals only overlap with the most immediate neighbors. As a consequence, the roll-off factor is equal to 1 , which implies that the summation zone in (3) solely encompasses the values $\{q-1, q, q+1\}$. It is important to mention that the effect of the channel does not widen the bandwidth occupied by the subcarrier signals. Therefore, the limitation done in formula (3), in terms of number of included summation elements, is accurate and does not have any influence on the performance of the receiver. The assumption that inter-carrier interference comes from the adjacent subcarriers would be wrong only in the presence of very high carrier frequency offsets. However, we assume that there is perfect synchronization between the transmitter and receiver, since we do not want to focus on the influence of the synchronization errors, rather our goal is to concentrate on the impact of multi-tap filtering on the transmit/noise power boost.

To illustrate the good spectral containment exhibited by the subcarrier signals, Figure 2 shows the power spectral density (PSD) of the signal transmitted on the tenth subchannel when the air interface is based on OFDM and FBMC/OQAM. The PSD is normalized so that the gain in the passband region is $0 \mathrm{~dB}$.

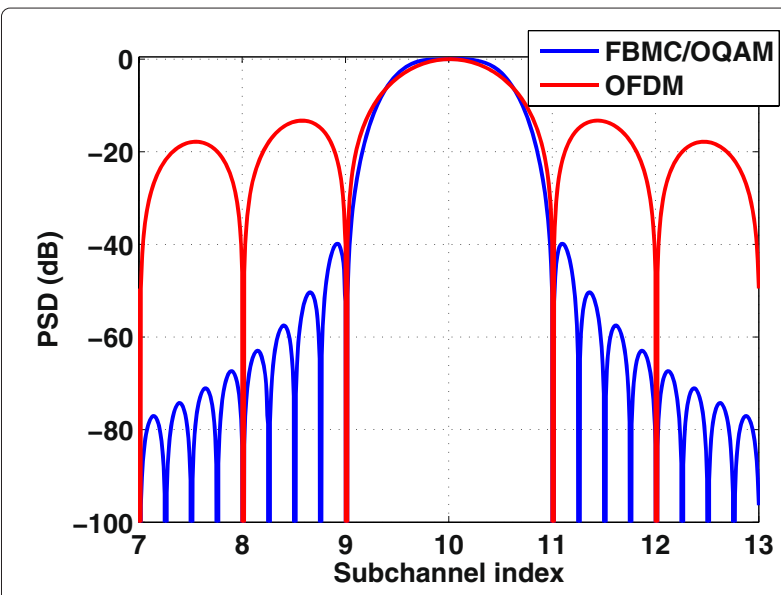

Figure 2 Power spectral density of the tenth subcarrier in OFDM and FBMC/OQAM systems.
Finally, the real-valued PAM symbols are estimated after compensating the phase term and extracting the real part, i.e., $\hat{d}_{q}[k]=\Re\left(\theta_{q}^{*}[k] \hat{x}_{q}[k]\right)$. After that, $\hat{d}_{q}[k]$ can be sent to the symbol demapper.

It is worth noticing that the joint design of precoders and equalizers is definitely challenging because the subcarriers are coupled. Therefore, the subcarriers have to be jointly optimized, which requires a complexity that becomes prohibitively high if $M$ is large. By applying some bounds, the problem can be simplified as it is demonstrated in [20], yet the complexity burden is still high. To alleviate the complexity, we concentrate on two simpler strategies to counteract the channel. The first one sets $a_{q}[k]=1$ for all $q$ and places the complexity at the transmit side. Then,

$$
\begin{aligned}
\hat{x}_{q}[k]= & \sum_{m=q-1}^{q+1}\left(d_{m}[k] \theta_{m}[k]\right) * b_{m}[k] * g_{q m}[k] \\
& +\left(v[n] * f_{q}^{*}[-n]\right)_{\downarrow \frac{M}{2}} .
\end{aligned}
$$

Alternatively, in the second approach, the system exclusively relies on the equalizers to combat the negative effects of the channel. Thus, (3) is simplified to

$$
\begin{aligned}
\hat{x}_{q}[k]= & \sum_{m=q-1}^{q+1} a_{q}[k] *\left(d_{m}[k] \theta_{m}[k]\right) * g_{q m}[k] \\
& +a_{q}[k] *\left(v[n] * f_{q}^{*}[-n]\right)_{\downarrow \frac{M}{2}},
\end{aligned}
$$

which is obtained by fixing $b_{m}[k]=1$ for all $m$.

\section{Subband processing}

Under ideal propagation conditions, that is in the absence of noise and multipath propagation, the symbols can be perfectly estimated as follows: $d_{q}[k]=\Re\left(\theta_{q}^{*}[k] y_{q}[k]\right)$, without resorting to neither precoders nor equalizers. However, $p[n]$ has to be designed to satisfy the perfect reconstruction property [5]. Based on this, it is clear that the objective of precoding and equalizing the symbols is to counteract the channel. In other words, the equivalent responses given by $b_{m}[k] * g_{q m}[k]$ and $a_{q}[k] * g_{q m}[k]$ should be as similar to $\left(f_{m}[n] * f_{q}^{*}[-n]\right)_{\downarrow \frac{M}{2}}$ as possible. For the sake of brevity, from here onwards, we exclusively focus on the precoding design, yet the same steps can be taken to design the equalizers. Regarding the optimization criterion, we have favored the design that is described in [15], which is based on the frequency sampling approach proposed in [9]. The rationale behind this choice has to do with the fact that the frequency sampling approach gives a satisfactory performance with affordable complexity. The objective pursued by this precoder is to set its frequency response to the given target values. In particular, we focus 
on the scheme that fixes the length of the precoders to be equal to 3 , i.e., $b_{m}[k] \neq 0$ for $-1 \leq k \leq 1$, because this configuration achieves the best trade-off between performance and complexity in most scenarios (see, e.g., $[8,9,15])$. As a consequence, the filter $b_{m}[k]$ is designed to equalize the channel at the following frequency points: $w_{m}^{(-1)}=\frac{2 \pi m}{M}-\frac{\pi}{M}, w_{m}^{(0)}=\frac{2 \pi m}{M}$, and $w_{m}^{(1)}=\frac{2 \pi m}{M}+\frac{\pi}{M}$. Without the loss of generality, precoders are expressed as the function of a finite impulse response filter that is multiplied by a scalar that controls the power allocated to each subband. In consequence, $b_{m}[k]=\sqrt{\alpha_{m}} u_{m}[k]$. Then, the following system of linear equations

$$
\begin{aligned}
\mathbf{C}_{e} \mathbf{u}_{m}= & {\left[\begin{array}{ccc}
e^{-j \frac{-\pi}{2}(-1)} & e^{-j \frac{-\pi}{2}(0)} & e^{-j \frac{\pi}{2}(1)} \\
e^{-j 0(-1)} & e^{-j 0(0)} & e^{-j 0(1)} \\
e^{-j \frac{\pi}{2}(-1)} & e^{-j \frac{\pi}{2}(0)} & e^{-j \frac{\pi}{2}(1)}
\end{array}\right]\left[\begin{array}{c}
u_{m}[-1] \\
u_{m}[0] \\
u_{m}[1]
\end{array}\right] } \\
= & {\left[\begin{array}{c}
\frac{H\left(w_{m}^{(-1)}\right)^{*}}{\left|H\left(w_{m}^{(-1)}\right)\right|^{2}+\eta} \\
\frac{H\left(w_{m}^{(0)}\right)^{*}}{\left|H\left(w_{m}^{(0)}\right)\right|^{2}+\eta} \\
\frac{H\left(w_{m}^{(1)}\right)^{*}}{\left|H\left(w_{m}^{(1)}\right)\right|^{2}+\eta}
\end{array}\right], }
\end{aligned}
$$

enables us to obtain transmit processing on even subbands. On odd subbands, the problem reads as follows:

$$
\begin{aligned}
\mathbf{C}_{o} \mathbf{u}_{m}= & {\left[\begin{array}{ccc}
e^{-j \frac{\pi}{2}(-1)} & e^{-j \frac{\pi}{2}(0)} & e^{-j \frac{\pi}{2}(1)} \\
e^{-j \pi(-1)} & e^{-j \pi(0)} & e^{-j \pi(1)} \\
e^{-j \frac{3 \pi}{2}(-1)} & e^{-j \frac{3 \pi}{2}(0)} & e^{-j \frac{3 \pi}{2}(1)}
\end{array}\right]\left[\begin{array}{c}
u_{m}[-1] \\
u_{m}[0] \\
u_{m}[1]
\end{array}\right] } \\
= & {\left[\begin{array}{c}
\frac{H\left(w_{m}^{(-1)}\right)^{*}}{\left|H\left(w_{m}^{(-1)}\right)\right|^{2}+\eta} \\
\frac{H\left(w_{m}^{(0)}\right)^{*}}{\left|H\left(w_{m}^{(0)}\right)\right|^{2}+\eta} \\
\frac{H\left(w_{m}^{(1)}\right)^{*}}{\left|H\left(w_{m}^{(1)}\right)\right|^{2}+\eta}
\end{array}\right] . }
\end{aligned}
$$

In notation terms, let $H(w)$ be the channel frequency response evaluated on the radial frequency $w$. The target points in (7) and (8), indicated explicitly by the right-hand side of these formulas, can be selected according to the zero forcing (ZF) or the mean square error (MSE) criteria. In this regard, we set $\eta=0$ when the $\mathrm{ZF}$ approach is followed. By contrast, the target points used in the MSE criterion are such that $\eta=N_{0}$. Finally, for both criteria, the vectors are scaled as follows: $\alpha_{m}=\frac{p_{m}}{\left\|\mathbf{u}_{m}\right\|_{2}^{2}}$. The power distribution can be designed to satisfy different criteria without violating the constraint $\sum_{m=0}^{M-1} p_{m} \leq P_{S}$, where $P_{S}$ denotes the maximum power that can be assigned to one multicarrier symbol.

When it comes to designing equalizers, the system of Equations in (7) and (8) can also be used to determine the value of each tap. Therefore, we obtain $a_{m}[k]=$ $\sqrt{\alpha_{m}} u_{m}[k]$. Now, the scaling factor is independent of the power distribution and is formulated as $\alpha_{m}=\frac{1}{\left\|\mathbf{u}_{m}\right\|_{2}^{2}}$.

\section{Detrimental effects of multi-tap filtering}

The perfect reconstruction property derived in [5] indicates that in the FBMC/OQAM context, the transmitted data can be perfectly recovered at the receive side under ideal propagation conditions. However, orthogonality properties are only satisfied in the real field. As a consequence, multipath propagation will certainly induce ISI and ICI. This justifies the need to counteract the channel in order to restore the orthogonality. As Section 2 proposes, signal processing techniques aimed at combating the channel can be performed either at the transmit or at the receive side. Previous works on this topic have concluded that multi-tap filtering becomes mandatory if the channel frequency selectivity is appreciable at the subcarrier level $[9,10,12-15]$. Nevertheless, the use of multiple taps may involve some detrimental effects. When broadband processing is applied at the transmitter, the power emitted might be boosted as if there was no precoding, because consecutive multicarrier symbols are correlated. By contrast, the consequence of performing multi-tap equalization is a possible noise enhancement. It is worth mentioning that in the FBMC/OQAM context, the power from the input of the modulator to the channel input is preserved if the transmit processing is based on singletap precoding. That is because the symbols transmitted in different subcarriers are independent along with the fact that $\sum_{\forall n}\left|f_{m}[n]\right|^{2}=1$ for all $m$, since the energy of the prototype pulse is equal to one. In the following, we will study the effects of multi-tap precoding and multitap equalization on the transmit and the noise power, respectively.

\subsection{Average transmit power}

To study the impact of precoding the symbols, we work with the most general model where the order of $\left\{b_{m}[k]\right\}$ is higher than 1. Considering that a frame transmission contains $N$ multicarrier symbols, the average transmit power per frame is given by

$$
\begin{aligned}
P_{\mathrm{T}}= & \sum_{n=0}^{L_{N}-1} \mathbb{E}\left\{\left|s_{\operatorname{tr}}[n]\right|^{2}\right\} \\
= & \sum_{n=0}^{L_{N}-1} \sum_{k, s=0}^{N-1} \sum_{m, l=0}^{M-1} \mathbb{E}\left\{( d _ { m } [ k ] \theta _ { m } [ k ] * b _ { m } [ k ] ) \left(d_{l}[s] \theta_{l}[s]\right.\right. \\
& \left.\left.* b_{l}[s]\right)^{*}\right\} f_{m}\left[n-k \frac{M}{2}\right] f_{l}^{*}\left[n-s \frac{M}{2}\right],
\end{aligned}
$$


where $L_{N}=(N-1) \frac{M}{2}+L$ accounts for the total number of samples. It is important to remark that the expectation is taken over symbols and precoders, which are assumed to be independent. If $\mathbb{E}\left\{d_{m}[k] d_{l}[s]\right\}=\delta_{m, l} \delta_{k, s}$, it follows that

$$
\begin{aligned}
\mathbb{E}\left\{\left|s_{\operatorname{tr}}[n]\right|^{2}\right\}= & \sum_{k, s=0}^{N-1} \sum_{m=0}^{M-1} \mathbb{E}\left\{\left(d_{m}[k] \theta_{m}[k] * b_{m}[k]\right)\right. \\
& \left.\times\left(d_{m}[s] \theta_{m}[s] * b_{m}[s]\right)^{*}\right\} f_{m}\left[n-k \frac{M}{2}\right] \\
& \times f_{m}^{*}\left[n-s \frac{M}{2}\right]
\end{aligned}
$$

with

$$
\begin{aligned}
& \mathbb{E}\left\{\left(d_{m}[k] \theta_{m}[k] * b_{m}[k]\right)\left(d_{m}[s] \theta_{m}[s] * b_{m}[s]\right)^{*}\right\} \\
& =\sum_{z, t=0}^{N-1} \mathbb{E}\left\{d_{m}[t] d_{m}[z] \theta_{m}[t] \theta_{m}^{*}[z] b_{m}[k-t] b_{m}^{*}[s-z]\right\} \\
& =\sum_{t=0}^{N-1} \mathbb{E}\left\{b_{m}[k-t] b_{m}^{*}[s-t]\right\} \\
& =\sum_{r=\max (-1, k-N+1)}^{\min (k, 1)} \mathbb{E}\left\{b_{m}[r] b_{m}^{*}[s+r-k]\right\} .
\end{aligned}
$$

It is worth mentioning that the variable $s$ in (10) cannot be omitted because the equality $\mathbb{E}$ $\left\{\left(d_{m}[k] \theta_{m}[k] * b_{m}[k]\right)\left(d_{m}[s] \theta_{m}[s] * b_{m}[s]\right)^{*}\right\}=p_{m} \delta_{k, s}$ is not satisfied. This can be verified by realizing that any two samples of the sequence $d_{m}[k] \theta_{m}[k] * b_{m}[k]$, which are evaluated in consecutive time epochs, are correlated. The term $s$ can only be omitted if precoders have a single tap. The summation zone in the last equality of (11) has been reduced since $b_{m}[k]$ is other than zero for $-1 \leq k \leq 1$.

According to (7) and (8), the terms $\left\{u_{m}[-1]\right.$, $\left.u_{m}[0], u_{m}[1]\right\}$ are function of the channel frequency response (CFR). Since it is customary to model the taps of the channel impulse response as random variables, the CFR evaluated at any given frequency is also a random variable, and therefore, the taps of the precoders are random variables that are uncorrelated with the symbols. Incorporating (10) and (11) into (9) results in

$$
\begin{aligned}
P_{\mathrm{T}}= & \sum_{n=0}^{L_{N}-1} \sum_{k, s=0}^{N-1} \sum_{m=0}^{M-1} \sum_{r=\max (-1, k-N+1)}^{\min (k, 1)} \mathbb{E}\left\{b_{m}[r] b_{m}^{*}[s+r-k]\right\} \\
& \times f_{m}\left[n-k \frac{M}{2}\right] f_{m}^{*}\left[n-s \frac{M}{2}\right] \\
= & \sum_{\substack{\min (k, 1) \\
N-1}} \sum_{m=0}^{M-1} \sum_{m=\max (-1, k-N+1)} \mathbb{E}\left\{b_{m}[r] b_{m}^{*}[s+r-k]\right\} \\
& \times R_{f_{m}}\left[(s-k) \frac{M}{2}\right]
\end{aligned}
$$

$$
=\sum_{\substack{k=0 \\ \text { ( }}}^{N-1} \sum_{\substack{z=k-N+1 \\ f_{m}}}^{k} \sum_{m=0}^{M-1} \sum_{r=\max (-1, k-N+1)}^{\min (k, 1)} \mathbb{E}\left\{b_{m}[r] b_{m}^{*}[r-z]\right\}
$$

with

$$
\begin{aligned}
R_{f_{m}}\left[(s-k) \frac{M}{2}\right] & =\sum_{\substack{n \in \mathbb{Z} \\
L-1+\min (s, k) \frac{M}{2}}} f_{m}\left[n-k \frac{M}{2}\right] f_{m}^{*}\left[n-s \frac{M}{2}\right] \\
& =\sum_{n=\max (s, k) \frac{M}{2}} p\left[n-s \frac{M}{2}\right] e^{j \pi m(s-k)} .
\end{aligned}
$$

From (12), it can be inferred that if precoders have a single tap, the average transmit power per frame will read as

$$
P_{\mathrm{T}}=\sum_{m=0}^{M-1} p_{m} N R_{f_{m}}[0] .
$$

In order to determine if the addressed subband processing leads to an increase of the transmit power, we shall compare (12) with (14). To simplify the analysis, we first split (12) into two terms as follows:

$$
P_{\mathrm{T}}=P_{\mathrm{T}_{1}}+P_{\mathrm{T}_{2}}
$$

$$
\begin{aligned}
z \neq 0 \rightarrow P_{\mathrm{T}_{1}}= & \sum_{k=0}^{N-1} \sum_{\substack{z=k-N+1 \\
z \neq 0}}^{k} \sum_{m=0}^{M-1} \sum_{r=\max (-1, k-N+1)}^{\min (k, 1)} \\
& \times \mathbb{E}\left\{b_{m}[r] b_{m}^{*}[r-z]\right\} R_{f_{m}}\left[-z \frac{M}{2}\right] \\
= & \sum_{k=0}^{N-1} \sum_{m=0}^{M-1} \mathbb{E}\left\{P_{m}^{1}[k]\right\}
\end{aligned}
$$

$$
\begin{aligned}
z=0 \rightarrow P_{\mathrm{T}_{2}} & =\sum_{k=0}^{N-1} \sum_{m=0}^{M-1} \sum_{r=\max (-1, k-N+1)}^{\min (k, 1)} \mathbb{E}\left\{\left|b_{m}[r]\right|^{2}\right\} R_{f_{m}}[0] \\
& =\sum_{k=0}^{N-1} \sum_{m=0}^{M-1} R_{f_{m}}[0] \mathbb{E}\left\{P_{m}^{2}[k]\right\}
\end{aligned}
$$

From (17), we can infer that $P_{\mathrm{T}_{2}}$ is always positive. By contrast, (16) may assume negative values, which indicates that $P_{\mathrm{T}_{1}}$ cannot be expressed in units of power. Since $P_{\mathrm{T}}$ accounts for the transmit power, it follows that $P_{\mathrm{T}} \geq 0$. Therefore, if $P_{\mathrm{T}_{1}}<0$, the inequality $\left|P_{\mathrm{T}_{1}}\right| \leq P_{\mathrm{T}_{2}}$ will always be satisfied. Taking into account that the $m$ th precoder has a norm equal to $\sqrt{p_{m}}$, then $P_{m}^{1}[k]$ and $P_{m}^{2}[k]$ can be expressed in the form of 


$$
\begin{aligned}
& P_{m}^{1}[k]= \begin{cases}b_{m}[0] b_{m}^{*}[1] R_{f_{m}}\left[\frac{M}{2}\right]+b_{m}[-1]\left(b_{m}^{*}[0] R_{f_{m}}\left[\frac{M}{2}\right]+b_{m}^{*}[1] R_{f_{m}}[M]\right) & k=0 \\
b_{m}[1] b_{m}^{*}[0] R_{f_{m}}\left[-\frac{M}{2}\right]+b_{m}[0] b_{m}^{*}[-1] R_{f_{m}}\left[-\frac{M}{2}\right]+P_{m}^{1}[0] & k=1 \\
b_{m}[1] b_{m}^{*}[-1] R_{f_{m}}[-M]+P_{m}^{1}[1] & 2 \leq k \leq N-3 \\
b_{m}[0] b_{m}^{*}[1] R_{f_{m}}\left[\frac{M}{2}\right]+b_{m}[-1] b_{m}^{*}[0] R_{f_{m}}\left[\frac{M}{2}\right]+P_{m}^{1}[N-1] & k=N-2 \\
b_{m}[1]\left(b_{m}^{*}[0] R_{f_{m}}\left[-\frac{M}{2}\right]+b_{m}^{*}[-1] R_{f_{m}}[-M]\right)+b_{m}[0] b_{m}^{*}[-1] R_{f_{m}}\left[-\frac{M}{2}\right] & k=N-1\end{cases} \\
& P_{m}^{2}[k]=\left\{\begin{array}{ll}
\left|b_{m}[-1]\right|^{2}+\left|b_{m}[0]\right|^{2} & k=0 \\
p_{m} & 1 \leq k \leq N-2 . \\
\left|b_{m}[0]\right|^{2}+\left|b_{m}[1]\right|^{2} & k=N-1
\end{array} .\right.
\end{aligned}
$$

Then, it follows that

$$
P_{\mathrm{T}_{2}}=\sum_{m=0}^{M-1}\left(\mathbb{E}\left\{\left|b_{m}[0]\right|^{2}\right\}+p_{m}(N-1)\right) R_{f_{m}}[0] .
$$

It is important to remark that the discrete orthogonality conditions restrict $R_{f_{m}}\left[-\frac{M}{2}\right], R_{f_{m}}\left[\frac{M}{2}\right]$ to be real-valued and $R_{f_{m}}[-M], R_{f_{m}}[M]$ to be pure imaginary [5]. Using the fact that $p[n]$ is real-valued and symmetric around the sample $n=\frac{L-1}{2}$, it can be deduced from (13) that $R_{f_{m}}\left[\frac{M}{2}\right], R_{f_{m}}\left[-\frac{M}{2}\right], R_{f_{m}}[-M], R_{f_{m}}[M] \in \mathbb{R}$. As a result, $R_{f_{m}}[M], R_{f_{m}}[-M]$ are only allowed to assume the value 0 . From (13), it can also be demonstrated that $R_{f_{m}}\left[\frac{M}{2}\right]=$ $R_{f_{m}}\left[-\frac{M}{2}\right]$ for all $m$, as well as $R_{f_{2 m}}\left[\frac{M}{2}\right]=-R_{f_{2 q-1}}\left[\frac{M}{2}\right]$ for $0 \leq m, q \leq \frac{M}{2}-1$. Bearing in mind the above discussion and assuming that the orthogonality properties are satisfied, $P_{\mathrm{T}_{1}}$ can be compactly formulated as

$$
\begin{aligned}
P_{\mathrm{T}_{1}}= & \sum_{m=0}^{M-1}(N-1) \mathbb{E}\left\{b_{m}[0] b_{m}^{*}[1]+b_{m}^{*}[0] b_{m}[1]\right. \\
& \left.+b_{m}[-1] b_{m}^{*}[0]+b_{m}^{*}[-1] b_{m}[0]\right\} R_{f_{m}}\left[\frac{M}{2}\right] .
\end{aligned}
$$

Note that $\left\{\left|u_{m}[-1]\right|_{2}^{2},\left|u_{m}[0]\right|_{2}^{2},\left|u_{m}[1]\right|_{2}^{2}\right\}$ are positive; thus, the maximum value that $\left|b_{m}[0]\right|^{2}$ can reach is lower or equal than $p_{m}$ for any channel realization. Then, $\mathbb{E}\left\{\left|b_{m}[0]\right|^{2}\right\} \leq p_{m}$, which implies that

$$
P_{\mathrm{T}_{2}} \leq \sum_{m=0}^{M-1} p_{m} N R_{f_{m}}[0] .
$$

Based on this result, the analysis of the transmit power hinges on the evaluation of the positivity of (21). Hence, if $P_{\mathrm{T}_{1}}<0$, we will conclude that there is no penalty for using the addressed transmit filters. On the contrary, if $P_{\mathrm{T}_{1}}>0$, we shall scale the symbols so that (14) is satisfied, which is equivalent to multiplying the symbols by $\beta=\sqrt{\frac{\sum_{m=0}^{M-1} p_{m} N R_{f_{m}}[0]}{P_{\mathrm{T}_{1}}+P_{\mathrm{T}_{2}}}}$. Taking into account (20) and (22), we can lower bound the scaling factor as follows: the expectation of a fraction cannot be computed straightforwardly. In this sense, Appendices 1 to 4 give details on how to obtain an approximate value of (21).

One alternative to compensate the possible boost of power due to multi-tap precoding is to evaluate (21) given the precoders, which is equivalent to dropping the expectation, and then using this value to compute $\beta$. While this ensures that the power is not increased, it entails the recalculation of $\beta$ whenever the precoder is modified. If we are able to characterize the expected value of $P_{\mathrm{T}_{1}}$, then $\beta$ does not have to be updated since its value is based on the statistical knowledge of the channel. The reduction of the complexity burden justifies the attempt to derive an analytical expression of (21).

\subsection{Average power of the equalized noise}

Analogously to the previous section, the average power of the equalized noise within one subband at the receiver side will be analyzed in this part. According to Figure 1, the received signal before the detection process can be described as

$$
\begin{aligned}
\hat{x}_{q}[k]= & a_{q}[k] * y_{q}[k]=D_{q}[k]+N_{q}[k] \\
= & \overbrace{\sum_{m=q-1}^{q+1}\left(d_{m}[k] \theta_{m}[k]\right) * b_{m}[k] * g_{q m}[k] * a_{q}[k]}^{\text {Desired part }} \\
& +\overbrace{\left(v[n] * f_{q}^{*}[-n]\right)_{\downarrow \frac{M}{2}} * a_{q}[k]}^{\text {Equalized noise }}
\end{aligned}
$$

where $D_{q}[k]$ refers to the desired part of the received signal, i.e., user data, and $N_{q}[k]=\left(v[n] * f_{q}^{*}[-n]\right)_{\downarrow \frac{M}{2}} * a_{q}[k]$ represents the colored (equalized) noise. Focusing on the second part of the formula, one can calculate the average noise power as $\mathbb{E}\left\{\left|N_{q}[k]\right|^{2}\right\}$. By denoting the first convolution in the noise term as $\zeta_{q}[k]$, i.e.,

$$
\zeta_{q}[k]=\left(v[n] * f_{q}^{*}[-n]\right)_{\downarrow \frac{M}{2}},
$$


the noise power in the qth subcarrier during the frame transmission can be represented as follows:

$P_{\mathrm{N}_{q}}=\sum_{k=0}^{N-1} \mathbb{E}\left\{\left|N_{q}[k]\right|^{2}\right\}=\sum_{k=0}^{N-1} \mathbb{E}\left\{\left(\zeta_{q}[k] * a_{q}[k]\right)\left(\zeta_{q}[k] * a_{q}[k]\right)^{*}\right\}$.

Taking into account that $\zeta_{q}[k] \neq 0$, for $0 \leq k \leq N-1$, one can rewrite formula (25) in this form:

$$
\begin{aligned}
P_{\mathrm{N}_{q}}= & \sum_{s, l=-1}^{0} \mathbb{E}\left\{a_{q}[l] a_{q}^{*}[s]\right\} \mathbb{E}\left\{\zeta_{q}[-l] \zeta_{q}^{*}[-s]\right\} \\
& +\sum_{s, l=0}^{1} \mathbb{E}\left\{a_{q}[l] a_{q}^{*}[s]\right\} \mathbb{E}\left\{\zeta_{q}[N-1-l] \zeta_{q}^{*}[N-1-s]\right\} \\
& +\sum_{k=1}^{N-2} \sum_{s, l=-1}^{1} \mathbb{E}\left\{a_{q}[l] a_{q}^{*}[s]\right\} \mathbb{E}\left\{\zeta_{q}[k-l] \zeta_{q}^{*}[k-s]\right\} .
\end{aligned}
$$

Taking into account relation (24), the expression $\mathbb{E}\left\{\zeta_{q}[k-l] \zeta_{q}^{*}[k-s]\right\}$ can be derived as

$$
\begin{aligned}
\mathbb{E}\left\{\zeta_{q}[k-l] \zeta_{q}^{*}[k-s]\right\}= & \mathbb{E}\left\{\sum_{u \in \mathbb{Z}} v[u] f_{q}^{*}\left[-(k-l) \frac{M}{2}+u\right]\right. \\
& \left.\times \sum_{r \in \mathbb{Z}} v^{*}[r] f_{q}\left[-(k-s) \frac{M}{2}+r\right]\right\} \\
= & \sum_{u, r \in \mathbb{Z}} \overbrace{\mathbb{E}\left\{v[u] v^{*}[r]\right\}}^{N_{0} \delta_{r, u}} \\
& \times \mathbb{E}\left\{f_{q}\left[-(k-s) \frac{M}{2}+r\right]\right. \\
& \left.\times f_{q}^{*}\left[-(k-l) \frac{M}{2}+u\right]\right\} \\
= & N_{0} \sum_{u \in \mathbb{Z}} f_{q}\left[-(k-s) \frac{M}{2}+u\right] \\
& \times f_{q}^{*}\left[-(k-l) \frac{M}{2}+u\right] .
\end{aligned}
$$

Substituting (27) to (26) and resorting to (13), the following relation is obtained:

$$
\begin{aligned}
P_{\mathrm{N}_{q}}= & N_{0} \sum_{l, s=-1}^{0} \mathbb{E}\left\{a_{q}[l] a_{q}^{*}[s]\right\} R_{f_{q}}\left[(s-l) \frac{M}{2}\right] \\
& +N_{0} \sum_{l, s=0}^{1} \mathbb{E}\left\{a_{q}[l] a_{q}^{*}[s]\right\} R_{f_{q}}\left[(s-l) \frac{M}{2}\right] \\
& +N_{0} \sum_{l, s=-1}^{1} \mathbb{E}\left\{a_{q}[l] a_{q}^{*}[s]\right\} R_{f_{q}}\left[(s-l) \frac{M}{2}\right] .
\end{aligned}
$$

Knowing that $a_{q}[k]=\sqrt{\alpha_{q}} u_{q}[k]$ as well as $R_{f_{q}}[M]=0$, it follows that $P_{\mathrm{N}_{q}}=P_{N_{q 1}}+P_{N_{q 2}}$, where

$$
\begin{aligned}
s=l \rightarrow P_{\mathrm{N}_{q 2}}= & \left(\mathbb{E}\left\{\left|a_{q}[0]\right|^{2}\right\}+\mathbb{E}\left\{\alpha_{q}\left\|\mathbf{u}_{q}\right\|_{2}^{2}\right\}(N-1)\right) \\
& \times N_{0} R_{f_{q}}[0] \leq \mathbb{E}\left\{\alpha_{q}\left\|\mathbf{u}_{q}\right\|_{2}^{2}\right\} N N_{0} R_{f_{q}}[0]
\end{aligned}
$$

$$
\begin{aligned}
s \neq l \rightarrow P_{\mathrm{N}_{q 1}}= & (N-1) N_{0} \mathbb{E}\left\{a_{q}[0] a_{q}^{*}[1]+a_{q}[1] a_{q}^{*}[0]\right. \\
& \left.+a_{q}[0] a_{q}^{*}[-1]+a_{q}[-1] a_{q}^{*}[0]\right\} R_{f_{q}}\left[\frac{M}{2}\right] .
\end{aligned}
$$

For the specific case of an one-tap equalizer, the above formula can be simplified as $P_{\mathrm{N}_{q}}=N N_{0} \mathbb{E}\left\{\left|a_{q}[0]\right|^{2}\right\} R_{f_{q}}$ [0]. If $b_{q}[k]=a_{q}[k], \forall q$, then we can state that the increase/decrease of the transmit power and the increase/decrease of the noise power for using multi-tap filtering coincide because this relation is satisfied

$$
\frac{P_{\mathrm{T}_{1}}+P_{\mathrm{T}_{2}}}{\sum_{q=0}^{M-1} p_{q} N R_{f_{q}}[0]}=\frac{\sum_{q=0}^{M-1} P_{\mathrm{N}_{q 1}}+P_{\mathrm{N}_{q 2}}}{\sum_{q=0}^{M-1} N N_{0} \mathbb{E}\left\{\left|a_{q}[0]\right|^{2}\right\} R_{f_{q}}[0]} .
$$

The denominator on the left-hand side corresponds to the transmit power, when precoders have a single tap. The denominator on the right-hand side accounts for the summation of the average noise power in all subcarriers, when the length of the equalizers is one. In light of condition (31), we may conclude that if symbols are properly scaled when the transmit power increases, then there is no degradation for counteracting the channel at reception rather than at transmission, as long as the same filters are used as precoders or equalizers.

\section{Numerical results}

This section provides some numerical results to provide insight into the effects of multi-tap filtering. In particular, we evaluate the average transmit power and the BER. As for the communication system, we simulate the model pictured in Figure 1. As it is proposed in Section 2, we concentrate on two simpler cases. The first scenario that is studied is based on a transmultiplexer that performs broadband filtering at the transmitter without equalizing the signals at reception. By contrast, the second scenario that is assessed is based on a system that exclusively hinges on multi-tap equalization to counteract the channel. Concerning the design criteria, we have favored the ZF approach described in Section 3. As Appendix 4 demonstrates, the ZF criterion offers better analytical tractability than the MSE alternative. Besides, 
the performance difference between ZF and MSE is small, as it is shown in [9]. As for the system parameters, the FBMC/OQAM modulation scheme splits the $10-\mathrm{MHz}$ bandwidth into $M=1,024$ or 512 subcarriers. The frame transmission comprises $N=20$ multicarrier symbols, and the sampling frequency is set to $f_{\mathrm{s}}=10 \mathrm{MHz}$ leading to subcarrier spacing equal to 9.76 or $19.53 \mathrm{KHz}$, depending on the total number of subcarriers. The channel is generated following the ITU Vehicular B (VehB) or the ITU Vehicular A (VehA) guidelines [21]. The transmitted symbols are generated by staggering real and imaginary parts of complex-valued symbols drawn from the 16-QAM constellation. It must be mentioned that the study of the impact that the power distribution may have on the results is out the scope of the paper, and thus, the power is equally split among subcarriers, i.e. $p_{m}=1 \forall m$.

\subsection{Average transmit power}

First, we study the impact of using the multiple-tap filters described in Section 3 as precoders on the average transmit power. To that end, $P_{\mathrm{T}_{1}}$ is approximately computed as detailed in Appendix 1. Since the proposed method is based on the knowledge of the statistical channel information (SCI), it will be identified from this point on with the acronym SCI. The SCI has been used to compute $P_{\mathrm{T}_{1}}$ as it is shown in Equations (41), (43), and (48). As a benchmark, we compute the empirical expectation of $P_{\mathrm{T}_{1}}$. This way of performing boils down to dropping the expectation in (21) and averaging $P_{\mathrm{T}_{1}}$ over 10,000 channel realizations. Then, $P_{\mathrm{T}_{1}}$ is the function of the precoders, which in turns are the function of the instantaneous CFR. Therefore, from here onwards, the acronym CFR will be used when we refer to the benchmark. To determine the validity of the proposed analytical expression, we gather in Table 1 the value of $P_{\mathrm{T}_{1}}$ that is obtained by SCI and CFR for different subcarrier spacing and channel models. The number of taps is set to three. The results confirm that the proposed method enables us to predict with a reasonable accuracy whether precoding results in an increased average transmitted power or not. The discrepancy between SCI and CFR is mostly due to the truncation of the Taylor series when computing (21). In any case, the difference is small with respect to $P_{\mathrm{T}_{2}}$ that is approximately equal to $M \times N=1,024 \times 20$ or $M \times N=512 \times 20$. The most interesting conclusion that can be drawn is that the precoding

Table 1 Computation of $P_{\mathrm{T}_{1}}$

\begin{tabular}{lcccc}
\hline & $\begin{array}{c}\boldsymbol{M}=\mathbf{1 , 0 2 4} \\
\text { (VehB) }\end{array}$ & $\begin{array}{c}\boldsymbol{M}=\mathbf{5 1 2} \\
\text { (VehB) }\end{array}$ & $\begin{array}{c}\boldsymbol{M}=\mathbf{1 , 0 2 4} \\
\text { (VehA) }\end{array}$ & $\begin{array}{c}\boldsymbol{M}=\mathbf{5 1 2} \\
\text { (VehA) }\end{array}$ \\
\hline SCI(frequency sampling) & -139.29 & -222.16 & -0.55 & -1.09 \\
CFR(frequency sampling) & -133.21 & -229.96 & -0.76 & -1.49 \\
CFR(MMSE) & 304.00 & 337.98 & -0.15 & 5.66 \\
\hline
\end{tabular}

design based on the frequency sampling approach comes at no cost in terms of the average transmit power since $P_{\mathrm{T}_{1}}$ is negative in all the cases.

In all the scenarios that have been simulated, it has been observed that multi-tap filtering has no harmful effects in terms of transmit power, thus supporting its utilization. In accordance with (31), we can also state that the average noise power is not enhanced due to multi-tap equalization. Although this result cannot be generalized for any channel, we provide the mathematical analysis to draw conclusions in other scenarios.

To determine if other techniques behave similarly to the frequency sampling approach, $P_{\mathrm{T}_{1}}$ has been computed when the taps of the precoders, i.e., $\left\{u_{m}[-1], u_{m}[0], u_{m}[1]\right\}$, are designed according to the MMSE criterion proposed in [10]. As Table 1 indicates, the MMSE precoder has a harmful effect in the average transmit power for VehB and VehA channels. However, for other types of channels, the conclusions may differ. It is left for future work to determine, in a mathematical or deductive way, which type of channels and precoders yield a positive $P_{\mathrm{T}_{1}}$.

\subsection{BER performance}

To show the importance of performing multi-tap precoding and equalization, we portray in Figures 3, 4, 5 and 6 the BER against the energy bit-to-noise ratio $\left(\frac{E_{b}}{N_{0}}\right)$ when the frequency sampling method is employed. Note that different subcarrier spacing and precoder and equalizer lengths have been simulated. In all figures, the curves corresponding to the systems that exclusively rely on precoding and those that rely on equalization virtually coincide. This observation is in line with the analysis conducted in Section 4. It must be highlighted that when the channel follows the VehB model, three-tap filtering

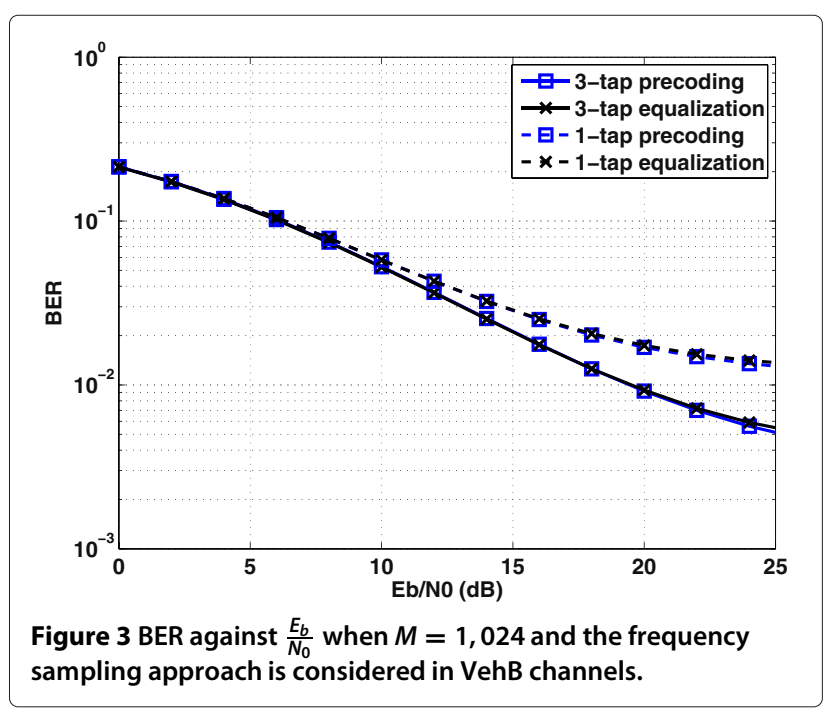




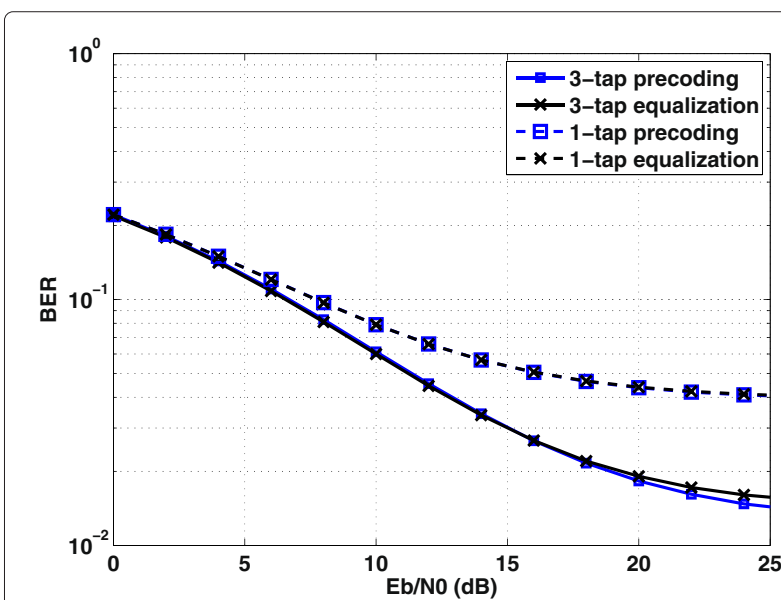

Figure 4 BER against $\frac{E_{b}}{N_{0}}$ when $M=512$ and the frequency sampling approach is considered in VehB channels. offers increased resilience against multipath fading when compared to the single-tap counterpart. This justifies the application of broadband processing. Nevertheless, the curves exhibit an error floor, which reveals that residual interference is present. As it is shown in [9], the BER deteriorates when the number of subcarriers is reduced, mainly because the subcarrier spacing widens. As a consequence, the equalization of the channel becomes more challenging, and so, the channel variations in the subcarrier pass band region may not be perfectly compensated. By examining Figures 5 and 6, we can conclude that when the propagation conditions are generated according to the VehA model, there is almost no degradation for neither reducing the number of subcarrier nor the number of taps. Therefore, we can assume that the channel frequency response is flat within the subchannels, even when

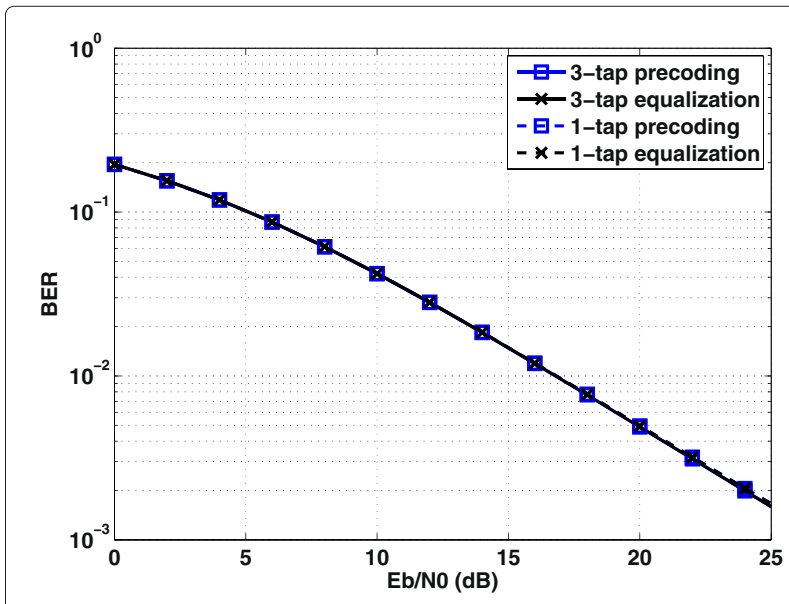

Figure 5 BER against $\frac{E_{b}}{N_{0}}$ when $M=1,024$ and the frequency sampling approach is considered in VehA channels.

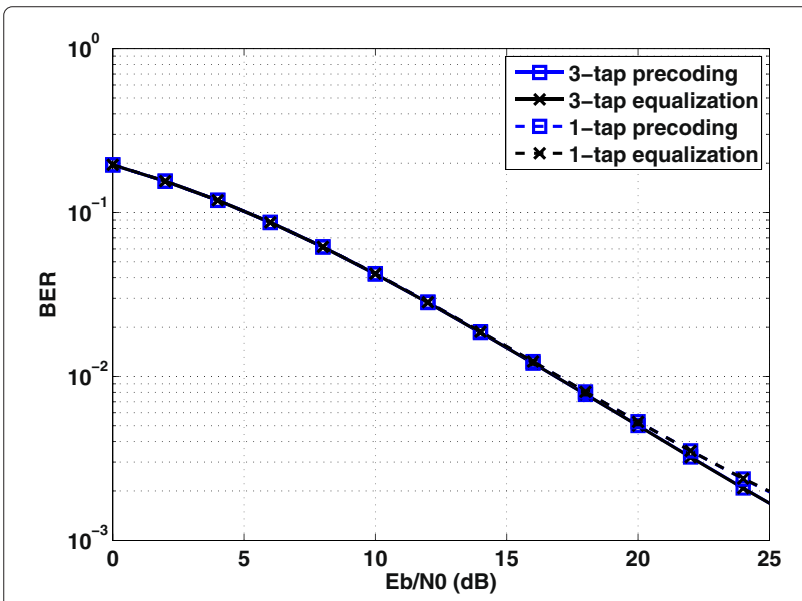

Figure 6 BER against $\frac{E_{b}}{N_{0}}$ when $M=512$ and the frequency sampling approach is considered in VehA channels.

$M=512$. Hence, in Figures 5 and 6, the channel is effectively compensated.

Note that the results depicted in Figures 3 and 4 have room for significant improvement. It is worth emphasizing that in our system, we do not use neither cyclic prefix nor channel coding; thus, the error floor will be significantly lowered, or will even disappear (see [22]), when the nowadays coders will be added. If bits are not encoded, then the error floor could be eliminated by applying a twostep approach as in $[11,13]$ or by performing a multi-band processing. However, the penalty that is paid to improve the system performance in both cases is an increased complexity burden, which may render the solution impractical. A less computationally demanding alternative, which is able to lower the BER to some extent, consists of designing precoders and equalizers according to the optimum MMSE criterion [10]. As the Figures 7 and 8 show, the optimum MMSE approach slightly outperforms the frequency sampling method in VehB channels. When the VehA model is simulated, the difference between the BER plots in Figures 5 and 6, and Figures 9 and 10 is almost non-existing. Therefore, we can state that the MMSE gives the best performance only at high $\frac{E_{b}}{N_{0}}$ in channels where the frequency selectivity is severe at the subcarrier level, which matches the conclusion drawn in [8]. It is worth noticing that the difference between the performance achieved for the frequency sampling method and the optimum MMSE criterion could be higher in the case of very low SNRs. In return, the complexity burden to compute the taps is substantially higher in the optimum MMSE case than in the frequency sampling counterpart (see [16]). The above discussion further motivates us to favorably consider the use of the frequency sampling method because it exhibits a good balance between complexity and performance. 


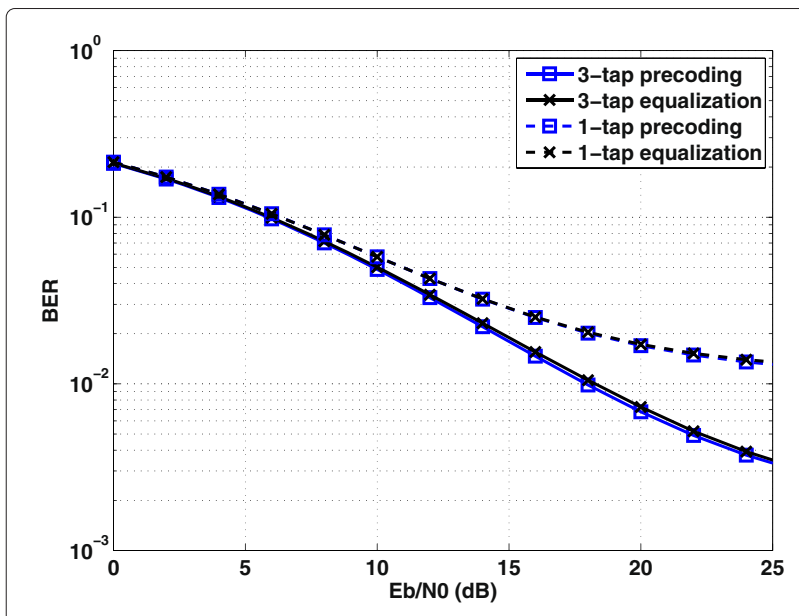

Figure 7 BER against $\frac{E_{b}}{N_{0}}$ when $M=1,024$ and the optimum MMSE criterion is considered in VehB channels.

Our current investigation confirms that there is still some space for improvement, mostly in highly frequencyselective channels, so finding a better trade-off between the performance and the complexity still remains as an open research problem in the FBMC/OQAM context. However, the goal of the paper is not to propose a new subband processing to combat the channel impairments with affordable complexity but to characterize the average transmit and noise power with the emphasis on systems with tractable complexity.

\section{Conclusions}

FBMC/OQAM has the key ingredients to deal with the restrictions that will be introduced by future wireless systems, such as the transmission in a fragmented spectrum. It is well known that the channel has to be counteracted

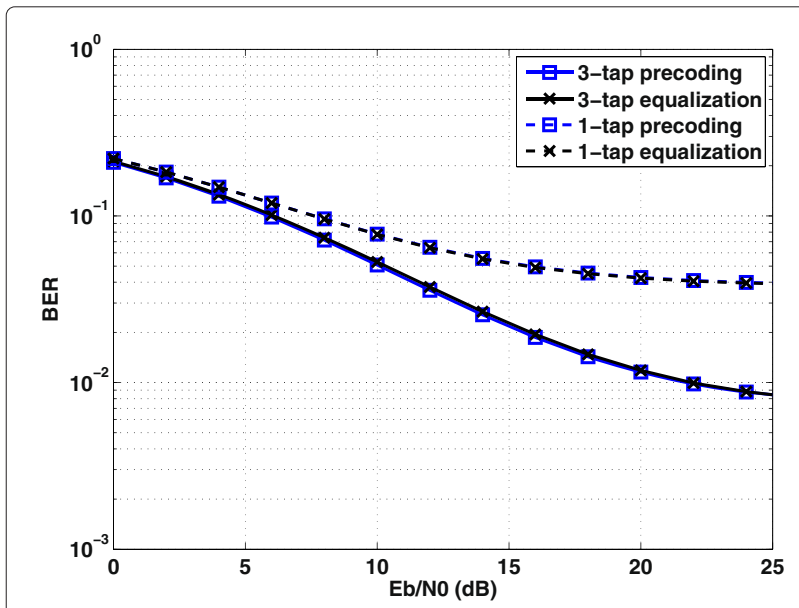

Figure 8 BER against $\frac{E_{b}}{N_{0}}$ when $M=512$ and the optimum MMSE criterion is considered in VehB channels.

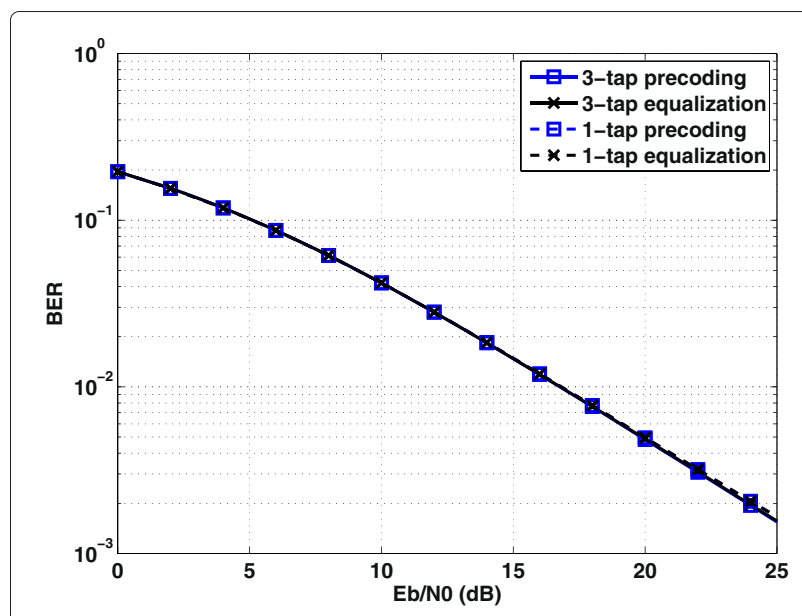

Figure 9 BER against $\frac{E_{b}}{N_{0}}$ when $M=1,024$ and the optimum MMSE criterion is considered in VehA channels.

to some extent in the FBMC/OQAM context to guarantee a certain quality of service. This translates into precoding the symbols at the transmit side or equalizing the demodulated data at the receive side. Both precoders and equalizers should perform multi-tap filtering when the channel is highly frequency-selective. The work presented here characterizes the average transmit power and the noise power when multi-tap precoders and equalizers are used. The closed-form expressions reveal that if the same filter is used as a precoder or equalizer, then the transmit power and the noise power increase or decrease with the same magnitude. Therefore, we can conclude that there is no degradation due to combating the channel at reception rather than at transmission, as long as the transmitted symbols are properly scaled if transmit processing boosts the power. To determine whether the

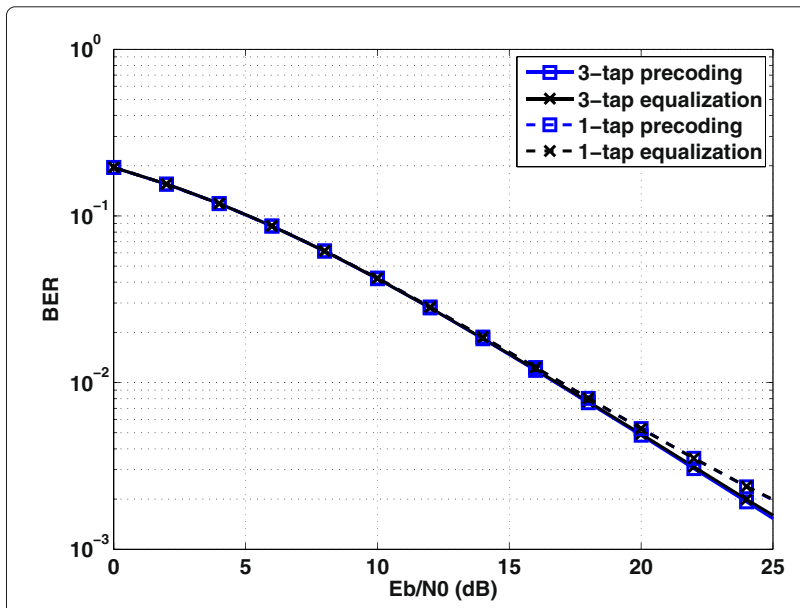

Figure 10 BER against $\frac{E_{b}}{N_{0}}$ when $M=512$ and the optimum MMSE criterion is considered in VehA channels. 
symbols should be scaled or not, we have formulated the transmit power as the function of the statistical knowledge of precoders when the criterion of design is based on the frequency sampling approach. The main reason to focus on the frequency sampling method is because it offers a good analytical tractability, which paves the way to get closed-form expressions, while it has a performance comparable to the optimum MMSE. The numerical results show that the analytical expressions derived in this paper are reasonably accurate, and thus, they can be used to address the issue related to the power boost. The alternative to using the precoder statistics consists in computing the instantaneous power. However, this solution is very demanding in terms of complexity, since the power has to be recalculated whenever precoders are updated. This highlights that the characterization of the transmit power derived in this paper, which relies on precoder statistics, is useful since it provides reliable information with reduced complexity. Both the numerical results and the closed-form expressions allow us to conclude that in the simulated scenarios, multi-tap precoding and equalization based on the frequency sampling approach do not boost the power. It is left for future work to investigate which precoding designs have a negative impact on the transmit power. Although the design of new equalization techniques is out of the scope of this paper, the numerical results have revealed that there is still space to improve the trade-off between complexity and performance of the state-of-the-art solutions.

\section{Appendices}

\section{Appendix 1: expectation of the ratio of two random variables}

In this appendix, we tackle how to approximately compute the statistical expectation of the ratio of two random variables, which is the key issue to determine the positivity of $P_{\mathrm{T}_{1}}$. Following the same steps described in [23], the expected value of $a / b$, where $a$ and $b$ are two correlated random variables, is given by

$$
\begin{aligned}
\mathbb{E}\left\{\frac{a}{b}\right\}= & \frac{\mathbb{E}\{a\}}{\mathbb{E}\{b\}} \mathbb{E}\left\{\left(1+\frac{b-\mathbb{E}\{b\}}{\mathbb{E}\{b\}}\right)^{-1}\right\} \\
& +\frac{1}{\mathbb{E}\{b\}} \mathbb{E}\left\{(a-\mathbb{E}\{a\})\left(1+\frac{b-\mathbb{E}\{b\}}{\mathbb{E}\{b\}}\right)^{-1}\right\}
\end{aligned}
$$

Let $b=b_{R}+j b_{I}$ and $\mathbb{E}\{b\}=\mu_{R}+j \mu_{I}$. Expanding $f\left(b_{R}, b_{I}\right)=\left(1+\frac{b_{R}-\mu_{R}+j\left(b_{I}-\mu_{I}\right)}{\mu_{R}+j \mu_{I}}\right)^{-1}$ as a Taylor series in $\left(\mu_{R}, \mu_{I}\right)$ leads to

$f\left(b_{R}, b_{I}\right)=\sum_{n_{1}, n_{2}=0}^{\infty} \frac{\left(b_{R}-\mu_{R}\right)^{n_{1}}\left(b_{I}-\mu_{I}\right)^{n_{2}}}{n_{1} ! n_{2} !}\left(\frac{\partial^{n_{1}+n_{2}} f}{\partial b_{R}^{n_{1}} \partial b_{I}^{n_{2}}}\right)\left(\mu_{R}, \mu_{I}\right)$.
It must be mentioned that we assume that the series converges, so that the equality between $f\left(b_{R}, b_{I}\right)$ and its Taylor series is valid. For complexity reasons, we have truncated the Taylor series so that only the terms $\left(n_{1}, n_{2}\right)=(0,0),\left(n_{1}, n_{2}\right)=(1,0)$, and $\left(n_{1}, n_{2}\right)=(0,1)$ have been considered. Then, $f\left(b_{R}, b_{I}\right)$ is given by

$$
f\left(b_{R}, b_{I}\right) \approx 1-\frac{b-\mathbb{E}\{b\}}{\mathbb{E}\{b\}} .
$$

Based on this result, $\mathbb{E}\left\{\frac{a}{b}\right\}$ can be approximated as follows:

$$
\mathbb{E}\left\{\frac{a}{b}\right\} \approx \frac{\mathbb{E}\left\{a\left(1-\frac{b-\mathbb{E}\{b\}}{\mathbb{E}\{b\}}\right)\right\}}{\mathbb{E}\{b\}} .
$$

Extending the series by including these terms $\left(n_{1}, n_{2}\right)=$ $(2,0),\left(n_{1}, n_{2}\right)=(0,2)$, and $\left(n_{1}, n_{2}\right)=(1,1)$, the function $f\left(b_{R}, b_{I}\right)$ becomes

$$
f\left(b_{R}, b_{I}\right) \approx 1-\frac{b-\mathbb{E}\{b\}}{\mathbb{E}\{b\}}+\frac{(b-\mathbb{E}\{b\})^{2}}{(\mathbb{E}(b))^{2}} .
$$

Then, $\mathbb{E}\left\{\frac{a}{b}\right\}$ can be written in this form

$$
\mathbb{E}\left\{\frac{a}{b}\right\} \approx \frac{\mathbb{E}\left\{a\left(1-\frac{b-\mathbb{E}\{b\}}{\mathbb{E}\{b\}}+\frac{(b-\mathbb{E}\{b\})^{2}}{(\mathbb{E}(b))^{2}}\right)\right\}}{\mathbb{E}\{b\}} .
$$

Although (37) is more accurate than (35), we will stick to the approximation written in (35), if otherwise stated. By extending the Taylor series, the function $f\left(b_{R}, b_{I}\right)$ is refined, but in exchange, the resulting expression is cumbersome. For this reason, we have favored (35) over (37), in some cases. In light of this discussion, we give priority to the analytical tractability by plugging $a=p_{m} u_{m}[s] u_{m}^{*}[z]$ and $b=\left\|\mathbf{u}_{m}\right\|_{2}^{2}$ into (35), which leads to this result

$$
\begin{aligned}
\mathbb{E}\left\{\frac{p_{m} u_{m}[s] u_{m}^{*}[z]}{\left\|\mathbf{u}_{m}\right\|_{2}^{2}}\right\} & \approx \frac{2 p_{m} \mathbb{E}\left\{u_{m}[s] u_{m}^{*}[z]\right\}}{\mathbb{E}\left\{\left\|\mathbf{u}_{m}\right\|_{2}^{2}\right\}} \\
& -\frac{p_{m} \mathbb{E}\left\{u_{m}[s] u_{m}^{*}[z]\left\|\mathbf{u}_{m}\right\|_{2}^{2}\right\}}{\left(\mathbb{E}\left\{\left\|\mathbf{u}_{m}\right\|_{2}^{2}\right\}\right)^{2}} .
\end{aligned}
$$

The coefficient $p_{m}$ can be taken out of the expectation as long as it is independent of the taps of the filter. From the expressions that are derived in Appendices 2 and 3, it is possible to infer how to compute (38), which enables us to evaluate (21) that is the ultimate goal. It is worth mentioning that the expressions provided in Appendices 2 and 3 are only valid when the subband processing is designed according to the ZF criterion. That is, when $\eta=0$ in (7) and (8). The analysis in the MSE case cannot be presented as concisely as in the ZF case. However, we indicate in Appendix 4 how to generalize the mathematical developments so that the MSE criterion is covered. 


\section{Appendix 2: computation of $\mathbb{E}\left\{u_{m}[s] u_{m}^{*}[z]\right\}$}

This appendix details how to obtain $\mathbb{E}\left\{u_{m}[s] u_{m}^{*}[z]\right\}$ for $m$ even and $s, z \in\{-1,0,1\}$. The process is identical for $m$ odd. Bearing in mind (7), the autocorrelation can be expressed as follows:

$$
\begin{aligned}
\mathbb{E}\left\{u_{m}[s] u_{m}^{*}[z]\right\}= & \sum_{x, y=-1}^{1}\left[\left(\mathbf{C}_{e}\right)^{-1}\right]_{(s+2)(x+2)}\left[\left(\mathbf{C}_{e}\right)^{-1}\right]_{(z+2)(y+2)}^{*} \\
& \times \mathbb{E}\left\{\frac{1}{H\left(w_{m}^{(x)}\right) H\left(w_{m}^{(y)}\right)^{*}}\right\}
\end{aligned}
$$

where $\left[\left(\mathbf{C}_{e}\right)^{-1}\right]_{a b}$ refers to the element of matrix $\left(\mathbf{C}_{e}\right)^{-1}$ located at the $a$ th row and $b$ th column. Note that we have to face again the problem of evaluating the expected value of a fraction. Resorting to (37), which is more accurate than (35), we can approximate $\mathbb{E}\left\{\frac{1}{H\left(w_{m}^{(x)}\right) H\left(w_{m}^{(y)}\right)^{*}}\right\}$ to

$$
\mathbb{E}\left\{\frac{1}{H\left(w_{m}^{(x)}\right) H\left(w_{m}^{(y)}\right)^{*}}\right\} \approx \frac{\mathbb{E}\left\{\left(H\left(w_{m}^{(x)}\right) H\left(w_{m}^{(y)}\right)^{*}\right)^{2}\right\}}{\left(\mathbb{E}\left\{H\left(w_{m}^{(x)}\right) H\left(w_{m}^{(y)}\right)^{*}\right\}\right)^{3}} .
$$

Step-by-step derivation of (40) hinges on the characterization of $\mathbb{E}\left\{H\left(w_{m}^{(x)}\right) H\left(w_{m}^{(y)}\right)^{*}\right\}$ and $\mathbb{E}\left\{\left(H\left(w_{m}^{(x)}\right) H\left(w_{m}^{(y)}\right)^{*}\right)^{2}\right\}$. The former expression is given by

$$
\mathbb{E}\left\{H\left(w_{m}^{(x)}\right) H\left(w_{m}^{(y)}\right)^{*}\right\}=\mathbb{E}\left\{\sum_{n, i=0}^{L_{h}-1} h[n] h^{*}[i] e^{-j w_{m}^{x} n} e^{j w_{m}^{y} i}\right\},
$$

where $h[n]$ denotes the channel impulse response. Assuming that the taps are modeled as independent complex Gaussian variables, i.e., $h[n] \sim \mathcal{C N}\left(0, \sigma_{n}^{2}\right)$, $\mathbb{E}\left\{h[n] h^{*}[i]\right\}=\delta_{n, i} \sigma_{n}^{2}$, Equation (41) can be transformed into

$$
\mathbb{E}\left\{H\left(w_{m}^{(x)}\right) H\left(w_{m}^{(y)}\right)^{*}\right\}=\sum_{n=0}^{L_{h}-1} \sigma_{n}^{2} e^{-j \frac{\pi}{M}(x-y) n}
$$

To complete the formulation of (40), we have to compute

$$
\begin{aligned}
\mathbb{E}\left\{\left(H\left(w_{m}^{(x)}\right) H\left(w_{m}^{(y)}\right)^{*}\right)^{2}\right\}= & \sum_{i, l, k, n=0}^{L_{h}-1} \mathbb{E}\left\{h[i] h^{*}[l] h[k] h^{*}[n]\right\} \\
& \times e^{-j w_{m}^{x} i} e^{j w_{m}^{y} l} e^{-j w_{m}^{x} k} e^{j w_{m}^{y} n} .
\end{aligned}
$$

Using the statistical channel information, it follows that

$\mathbb{E}\left\{h[i] h^{*}[l] h[k] h^{*}[n]\right\}=\left\{\begin{array}{ll}2 \sigma_{i}^{4} & i=l=k=n \\ \sigma_{i}^{2} \sigma_{n}^{2} & i=l, n=k, i \neq n \\ \sigma_{i}^{2} \sigma_{l}^{2} & i=n, l=k, i \neq l \\ 0 & \text { otherwise }\end{array}\right.$.

Plugging (44) into (43) leads to

$$
\begin{aligned}
& \mathbb{E}\left\{H\left(w_{m}^{(x)}\right) H\left(w_{m}^{(y)}\right)^{*} H\left(w_{m}^{(x)}\right) H\left(w_{m}^{(y)}\right)^{*}\right\} \\
& =\sum_{i=0}^{L_{h}-1} 2 \sigma_{i}^{4} e^{-j \frac{\pi}{M}(2 x-2 y) i} \\
& \quad+\sum_{i=0}^{L_{h}-1} \sum_{n=0, n \neq i}^{L_{h}-1} \sigma_{i}^{2} \sigma_{n}^{2} e^{-j \frac{\pi}{M}(x-y) i} e^{-j \frac{\pi}{M}(x-y) n} \\
& \quad+\sum_{i=0}^{L_{h}-1} \sum_{l=0, l \neq i}^{L_{h}-1} \sigma_{i}^{2} \sigma_{l}^{2} e^{-j \frac{\pi}{M}(x-y) i} e^{-j \frac{\pi}{M}(x-y) l} .
\end{aligned}
$$

With (45) and (42), which are independent of $m$, we can obtain an approximated value of (39) as function of the statistics of the channel.

Appendix 3: computation of $\mathbb{E}\left\{u_{m}[s] u_{m}^{*}[z]\left|u_{m}[l]\right|^{2}\right\}$ Similarly to Appendix 2, we start by formulating $\mathbb{E}\left\{u_{m}[s] u_{m}^{*}[z]\left|u_{m}[l]\right|^{2}\right\}$ as function of the channel frequency response for $m$ even and $s, z, l \in\{-1,0,1\}$. In this sense, we obtain

$$
\begin{aligned}
\mathbb{E}\left\{u_{m}[s] u_{m}^{*}[z]\left|u_{m}[l]\right|^{2}\right\}= & \sum_{r, t, x, y=-1}^{1}\left[\left(\mathbf{C}_{e}\right)^{-1}\right]_{(s+2)(r+2)}\left[\left(\mathbf{C}_{e}\right)^{-1}\right]_{(z+2)(t+2)}^{*}\left[\left(\mathbf{C}_{e}\right)^{-1}\right]_{(l+2)(x+2)} \\
& \times\left[\left(\mathbf{C}_{e}\right)^{-1}\right]_{(l+2)(y+2)}^{*} \mathbb{E}\left\{\frac{1}{H\left(w_{m}^{(r)}\right) H\left(w_{m}^{(t)}\right)^{*} H\left(w_{m}^{(x)}\right) H\left(w_{m}^{(y)}\right)^{*}}\right\} .
\end{aligned}
$$


Considering model (37), we can characterize (46) using this approximation

$$
\mathbb{E}\left\{\frac{1}{H\left(w_{m}^{(r)}\right) H\left(w_{m}^{(t)}\right)^{*} H\left(w_{m}^{(x)}\right) H\left(w_{m}^{(y)}\right)^{*}}\right\} \approx \frac{\mathbb{E}\left\{\left(H\left(w_{m}^{(r)}\right) H\left(w_{m}^{(t)}\right)^{*} H\left(w_{m}^{(x)}\right) H\left(w_{m}^{(y)}\right)^{*}\right)^{2}\right\}}{\left(\mathbb{E}\left\{H\left(w_{m}^{(r)}\right) H\left(w_{m}^{(t)}\right)^{*} H\left(w_{m}^{(x)}\right) H\left(w_{m}^{(y)}\right)^{*}\right\}\right)^{3}} .
$$

The denominator of the right-hand side of (47) can be obtained from the expressions written in (44) and (45). The numerator can be expressed as function of the channel impulse response as follows:

$$
\begin{aligned}
\mathbb{E}\left\{\left(H\left(w_{m}^{(r)}\right) H\left(w_{m}^{(t)}\right)^{*} H\left(w_{m}^{(x)}\right) H\left(w_{m}^{(y)}\right)^{*}\right)^{2}\right\}= & \sum_{\substack{i, i^{\prime}, l, l^{\prime}=0 \\
k, k^{\prime}, n, h^{\prime}=0}}^{L_{h}-1} \mathbb{E}\left\{h[i] h\left[i^{\prime}\right] h^{*}[l] h^{*}\left[l^{\prime}\right] h[k] h\left[k^{\prime}\right] h^{*}[n] h^{*}\left[n^{\prime}\right]\right\} \\
& \times e^{-j w m_{m}^{\prime}} e^{-j w_{m}^{r} i^{\prime}} e^{j w_{m}^{t} l} e^{j w_{m}^{t} l^{\prime}} e^{-j w_{m}^{x} k} e^{-j w_{m}^{x} k^{\prime}} \\
& \times e^{j w_{m}^{\prime} n} e^{j w_{m}^{y} n^{\prime}} .
\end{aligned}
$$

To formulate (48) as function of the channel statistics, it is necessary to compute $\mathbb{E}\left\{h[i] h\left[i^{\prime}\right] \cdots h^{*}[n] h^{*}\left[n^{\prime}\right]\right\}$. In this sense, we can follow the approach presented in [24] to characterize the expectation as function of $\left\{\sigma_{n}^{2}\right\},\left\{\sigma_{n}^{4}\right\},\left\{\sigma_{n}^{6}\right\}$ and $\left\{\sigma_{n}^{8}\right\}$, depending on the value of $i, i^{\prime}, l, l^{\prime}, k, k^{\prime}, n, n^{\prime}$. Since there are a lot of combinations that result in different expressions and thus a lot of space will be needed, we have not included the closed-form expression of (48). However, the steps required to compute (48) using the statistics of the channel are indicated, which allows us to approximately compute (46). Note that again, the result will be constant regardless of the subcarrier index.

\section{Appendix 4: comments on the MSE approach}

In this appendix, we would like to show that the approach proposed in details for ZF can be also applied for MSE criterion; however, the mathematical tractability is highly demanding in that case. Since the main difference between the ZF and the MSE concerns the definition of the target points, the whole derivations applied for ZF criterion can be straightforwardly used for MSE until (38). From that point on, some additional comments have to be made in order to tackle the computation of $\mathbb{E}\left\{u_{m}[s] u_{m}^{*}[z]\right\}$ and $\mathbb{E}\left\{u_{m}[s] u_{m}^{*}[z]\left|u_{m}[l]\right|^{2}\right\}$. Focusing first on the former one, we get

$$
\begin{aligned}
\mathbb{E}\left\{u_{m}[s] u_{m}^{*}[z]\right\}= & \sum_{x, y=-1}^{1}\left[\left(\mathbf{C}_{e}\right)^{-1}\right]_{(s+2)(x+2)}\left[\left(\mathbf{C}_{e}\right)^{-1}\right]_{(z+2)(y+2)}^{*} \\
& \times \mathbb{E}\left\{\frac{H\left(w_{m}^{(x)}\right)^{*} H\left(w_{m}^{(y)}\right)}{\left(\left|H\left(w_{m}^{(x)}\right)\right|^{2}+\eta\right)\left(\left|H\left(w_{m}^{(y)}\right)\right|^{2}+\eta\right)^{*}}\right\} .
\end{aligned}
$$

Analogously, the latter expectation can be represented as

$$
\begin{aligned}
& \mathbb{E}\left\{u_{m}[s] u_{m}^{*}[z]\left|u_{m}[l]\right|^{2}\right\}= \\
& \sum_{r, t, x, y=-1}^{1}\left[\left(\mathbf{C}_{e}\right)^{-1}\right]_{(s+2)(r+2)}\left[\left(\mathbf{C}_{e}\right)^{-1}\right]_{(z+2)(t+2)}^{*}\left[\left(\mathbf{C}_{e}\right)^{-1}\right]_{(l+2)(x+2)}\left[\left(\mathbf{C}_{e}\right)^{-1}\right]_{(l+2)(y+2)}^{*} \\
& \times \mathbb{E}\left\{\frac{H\left(w_{m}^{(r)}\right)^{*} H\left(w_{m}^{(t)}\right) H\left(w_{m}^{(x)}\right)^{*} H\left(w_{m}^{(y)}\right)}{\left(\left|H\left(w_{m}^{(r)}\right)\right|^{2}+\eta\right)\left(\left|H\left(w_{m}^{(t)}\right)\right|^{2}+\eta\right)^{*}\left(\left|H\left(w_{m}^{(x)}\right)\right|^{2}+\eta\right)\left(\left|H\left(w_{m}^{(y)}\right)\right|^{2}+\eta\right)^{*}}\right\} .
\end{aligned}
$$

In both cases, the expected value of the division of random variables can be approximated according to (35) and (37). Assuming the channel model introduced in Appendix 2, it is easily to observe that the derivation of closed-form formulas for MSE require calculation of $n$th order moments of Gaussian variables, which, in general form, can be represented as

$$
\mu_{r_{1}, \ldots, r_{m}}(Z)=\mathbb{E}\left\{\prod_{i=1}^{m} Z_{i}^{r_{i}}\right\}
$$


where $r_{1}+r_{2}+\ldots+r_{m}=n, r_{i} \geq 1$, and $\left\{Z_{i}\right\}$ are complexvalued random variables. The effective and generic way to calculate the expectation of the product of $m$ complex random variables is presented in [24], particularly in Definition 1 and the succeeding formula (5). Following that approach, one can calculate the closed-form for MSE criteria, and, in general,- for any other criteria which could be presented in the form discussed in that paper.

\section{Competing interests}

The authors declare that they have no competing interests.

\section{Acknowledgments}

This work has received funding from the Spanish Ministry of Economy and Competitiveness (Ministerio de Economia y Competitividad) under project TEC2011-29006-C03-02 (GRE3N-LINK-MAC) and from the Catalan Government (2009SGR0891). This work was also supported by the European Commission in the framework of the FP7 Network of Excellence in Wireless COMmunications NEWCOM\# (Grant agreement no. 318306).

\section{Author details}

${ }^{1}$ Department of Signal Theory and Communications, Universitat Politecnica de Catalunya (UPC), Barcelona 08034, Spain. ${ }^{2}$ Centre Tecnologic de Telecomunicacions de Catalunya (CTTC), Castelldefels, Barcelona 08860, Spain. ${ }^{3}$ Chair of Wireless Communications, Poznan University of Technology (PUT), Poznan 60-965, Poland.

\section{Received: 2 December 2013 Accepted: 19 May 2014}

\section{Published: 5 June 2014}

\section{References}

1. B Farhang-Boroujeny, R Kempter, Multicarrier communication techniques for spectrum sensing and communication in cognitive radios. IEEE Comm. Mag. 46(4), 80-85 (2008)

2. B Farhang-Boroujeny, OFDM versus filter bank multicarrier. IEEE Signal Process. Mag. 28(3), 92-112 (2011)

3. ICT-318362 Project EMPHATIC, Deliverable 2.1: FB-MC and enhanced OFDM schemes (2013). http://www.ict-emphatic.eu/. Accessed 01 Nov 2013

4. ICT-317669 Project METIS, Deliverable 2.1: requirements and general design principles for new air interface (2013). https://www.metis2020. com/documents/deliverables/. Accessed 01 Nov 2013

5. P Siohan, C Siclet, N Lacaille, Analysis and design of OFDM/OQAM systems based on filterbank theory. IEEE Trans. Signal Process. 50(5), 1170-1183 (2002)

6. HG Feichtinger, T Strohmer, Advances in Gabor Analysis. (Birkhauser, Boston, 2002)

7. J Yli-Kaakinen, M Renfors, Fast-convolution filter bank approach for non-contiguous spectrum use. Paper presented at the future network and mobile summit (FutureNetworkSummit) Lisbon, Portugal, 03-05 July 2013, pp. 1-10

8. PHYDYAS ICT-211887 Project, Deliverable 3.1: equalization and demodulation in the receiver (single antenna) (2008). http://www.ictphydyas.org/delivrables/. Accessed 01 Nov 2013

9. T Ihalainen, TH Stitz, M Rinne, M Renfors, Channel equalization in filter bank based multicarrier modulation for wireless communications. EURASIP J. Appl. Signal Process. 2007, 049389 (2007). doi:10.1155/2007/49389

10. DS Waldhauser, LG Baltar, J Nossek, MMSE subcarrier equalization for filter bank based multicarrier systems. Paper presented at the IEEE 9th workshop on signal processing advances in wireless communications (SPAWC), Recife, Brazil, 6-9 July 2008, pp. 525-529

11. H Lin, C Lele, P Siohan, Equalization with interference cancellation for Hermitian symmetric OFDM/OQAM systems. Paper presented at the IEEE international symposium on power line communications and its applications (ISPLC), Jeju Island, Korea, 2-4 April 2008, pp. 363-368

12. LG Baltar, DS Waldhauser, JA Nossek, MMSE subchannel decision feedback equalization for filter bank based multicarrier systems. Paper presented at the IEEE international symposium on circuits and systems (ISCAS), Taipei, Taiwan, 24-27 May 2009, pp. 2802-2805

13. A Ikhlef, J Louveaux, An enhanced MMSE per subchannel equalizer for highly frequency selective channels for FBMC/OQAM systems. Paper presented at the IEEE 10th workshop on signal processing advances in wireless communications (SPAWC), Marrakech, Morocco, 20-23 June 2010, pp. 186-190

14. M Caus, A Perez-Neira, Space-time receiver for filterbank based multicarrier systems. Paper presented at the international ITG workshop on smart antennas (WSA), Bremen, Germany, 23-24 Feb 2010, pp. $421-427$

15. M Caus, Al Perez-Neira, Transmit and receive filters for MISO FBMC systems subjected to power constraints. Paper presented in IEEE international conference on acoustics, speech and signal processing (ICASSP), Prague, Czech Republic, 22-27 May 2011, pp. 2660-2663

16. T Ihalainen, A Ikhlef, J Louveaux, M Renfors, Channel equalization for multi-antenna FBMC/OQAM receivers. IEEE Trans. Veh. Tech. 60(5), 2070-2085 (2011). IEEE

17. M Joham, W Utschick, JA Nossek, Linear transmit processing in MIMO communications systems. IEEE Trans. Signal Process. 53(8), 2700-2712 (2005)

18. D Kong, D Qu, T Jiang, Time domain channel estimation for OQAM-OFDM systems: algorithms and performance bounds. IEEE Trans. Signal Process. 62(2), 322-330 (2014)

19. MG Bellanger, Specification and design of a prototype filter for filter bank based multicarrier transmission. Paper presented at the IEEE international conference on acoustics, speech, and signal processing (ICASSP), vol. 4, Salt Lake City, UT, USA, 7-11 May 2001, pp. 2417-2420

20. M Caus, Al Perez-Neira, Transmitter-receiver designs for highly frequency selective channels in MIMO FBMC systems. IEEE Trans. Signal Process. 60(12), 6519-6532 (2012)

21. International Mobile Telecommunications, ITU-R, Guidelines for the evaluation of radio transmission technologies for IMT-2000. Recommendation ITU-R M.1225. (ITU, 1997). http://www.itu.int/rec/RREC-M.1225/en

22. LG Baltar, A Mezghani, JA Nossek, MLSE and MMSE subchannel equalization for filter bank based multicarrier systems: coded and uncoded results. Paper presented at European signal processing conference (EUSIPCO), Aalborg, Denmark, 23-27 Aug 2010, pp. $2186-2190$

23. S Rice, A stochastic version of the price equation reveals the interplay of deterministic and stochastic processes in evolution. BMC Evol. Biol. 8(1), 262 (2008)

24. KTriantafyllopoulosi, Moments and Cumulants of the Multivariate Real and Complex Gaussian Distributions, Version 12. (Department of Mathematics, University of Bristol, Bristol, 2002)

doi:10.1186/1687-6180-2014-84

Cite this article as: Caus et al:: Characterization of the effects of multi-tap filtering on FBMC/OQAM systems. EURASIP Journal on Advances in Signal Processing 2014 2014:84.

\section{Submit your manuscript to a SpringerOpen ${ }^{\odot}$ journal and benefit from:}

- Convenient online submission

Rigorous peer review

- Immediate publication on acceptance

- Open access: articles freely available online

- High visibility within the field

- Retaining the copyright to your article

Submit your next manuscript at springeropen.com 